\title{
Fast Temporal Dynamics of Visual Cue Integration
}

\author{
Jochen Triesch, Dana H. Ballard and Robert A. Jacobs \\ The University of Rochester \\ Computer Science Department \\ Rochester, New York 14627
}

Technical Report 00.2

September 2000

This work was supported by NIH/PHS research grant P41 RR09283 and by NIH research grants R29-MH54770 and RO1-EY13149. 


\begin{abstract}
We study the dynamics of visual cue integration in a tracking / identification task, where subjects track a target object among distractors and identify the target after an occlusion. Objects are defined by three different attributes (color, shape, size) which change randomly within a singe trial. When the attributes differ in their reliability (two change frequently, one is stable) our results show that subjects rapidly re-weight the different cues putting more emphasis on the stable cue. The re-weighting takes place in less than one second. Our results suggest that cue integration can exhibit adaptive phenomena on a very fast time scale. We propose a probabilistic model with temporal dynamics that accounts for the observed effect.
\end{abstract}

\title{
1 Introduction
}

A fundamental question in neuroscience is how the brain integrates information derived from different cues or modalities into coherent percepts. Many studies have looked at this question from behavioral, computational, and neurophysiological viewpoints. Unfortunately, different integration strategies have been observed in different experiments. Examples are weighted averaging (von Holst, 1950; Bruno \& Cutting, 1988; Landy, Maloney, Johnston \& Young, 1995), multiplicative interactions (Stein \& Meredith, 1993), boolean logic (Newman \& Hartline, 1982), fuzzy logic (Massaro \& Friedman, 1990), and linear and nonlinear Bayesian inference (Yuille \& Bülthoff, 1996). It seems that the way in which different cues are integrated depends on the cues involved, the nature of the task, characteristics of the sensory environment, and prior experience and knowledge of the observers. We believe that an important reason that investigators have been unable to identify the "cue integration strategy" is that observers do not use a single, immutable strategy. Rather, they use a collection of context-sensitive strategies that are adaptable in an experience-dependent manner.

Evidence of the adaptability of cue integration strategies has been reported earlier (von Holst, 1950), but has recently begun to accumulate. Jacobs \& Fine (1999) used a cueconflict experimental paradigm to show that observers' cue combination strategies for visual depth are adaptable as a function of training; subjects adjusted their cue combination rules to use a visual cue more heavily after training in which that cue was informative versus after training in which the cue was irrelevant. Moreover, these researchers showed that observers can learn multiple cue combination rules, and can learn to apply each rule in its appropriate context. Ernst, Banks \& Bülthoff (2000) studied the adaptability of observers' cue integration strategies using a virtual-reality environment that allowed subjects to interact with viewed objects by touching them. They showed that subjects' estimates of visual slant relied more heavily on a visual cue when the cue was consistent with haptic feedback versus when it was inconsistent with this feedback. Also using a virtual-reality environment, Atkins, Fiser \& Jacobs (2000) showed that observers compare visual and haptic percepts in order to evaluate the relative reliabilities of visual cues, and use these reliabilities in order to determine how to combine cues during three-dimensional visual perception. In addition, observers are 
able to learn multiple, context-sensitive cue integration strategies by comparing visual and haptic percepts.

Although it now seems clear that observers' visual cue integration strategies are adaptable, little is known about this adaptation process. For example, the studies reviewed above demonstrate adaptation on a relatively long time-scale (hours or days). However, they do not address whether or not this adaptation can influence on-line processing by modifying observers' cue integration rules on a short time-scale (fractions of a second). This article studies the fast temporal dynamics of visual cue integration by reporting the results of an experiment using a tracking / identification task. Subjects tracked a target object among distractors and identified the target after an occlusion. Objects were defined by three visual attributes (color, shape, and size). In each trial two of the attributes were unreliable in the sense that their values changed frequently within a trial, whereas the remaining attribute was reliable, i.e. its value did not change. The results are that subjects rapidly re-weighted the different cues on each trial by emphasizing the information provided by the reliable cue and by discounting the information provided by the unreliable cues. This re-weighting took place in less than one second and, thus, the results show that cue integration can exhibit adaptive phenomenon on a very fast time-scale. The experimental results are successfully accounted for by a probabilistic model with temporal dynamics.

\section{General methods}

Stimuli and apparatus; The experiment was performed using a virtual reality apparatus (Pelz, Hayhoe, Ballard, Shrivastava, Bayliss \& von der Heyde, 1999). A visual environment was rendered by a Silicon Graphics workstation on a pair of displays embedded in head-mounted goggles (see Figure 1). The environment consisted of twelve virtual objects. Objects were defined by shape, color, and size attributes. Each attribute could take one of two possible values. The possible object shape values were sphere and cube, the possible color values were yellow and green, and the possible size values were small $(2 \mathrm{~cm})$ and big $(5 \mathrm{~cm})$. Subjects freely adjusted their viewing distance to the objects by altering their seating position. The average distance was $69 \pm 9 \mathrm{~cm}$, meaning that the average visual angle of small and large objects was 1.7 and 4.1 degrees, respectively.

Objects moved in a square region of the frontoparallel plane. Each side of the region was $40 \mathrm{~cm}$ in length. The initial velocity of an object was selected at random (mean= $24.5 \mathrm{~cm} / \mathrm{sec}$; for the typical viewing distance, this corresponds to an angular velocity of $19.5 \mathrm{deg} / \mathrm{sec}$ ). Objects bounced when they collided with the sides of the square region or with each other. Because objects exchanged energy when they collided according to the laws of Newtonian physics, their velocities changed during the course of a trial but the total energy of the system remained constant. For the sake of simplicity, all objects were given equal masses.

Procedure: A subject's task was to visually track one of the objects and to identify it after an artificial occlusion. At the start of each trial, two choices were made. First, one 

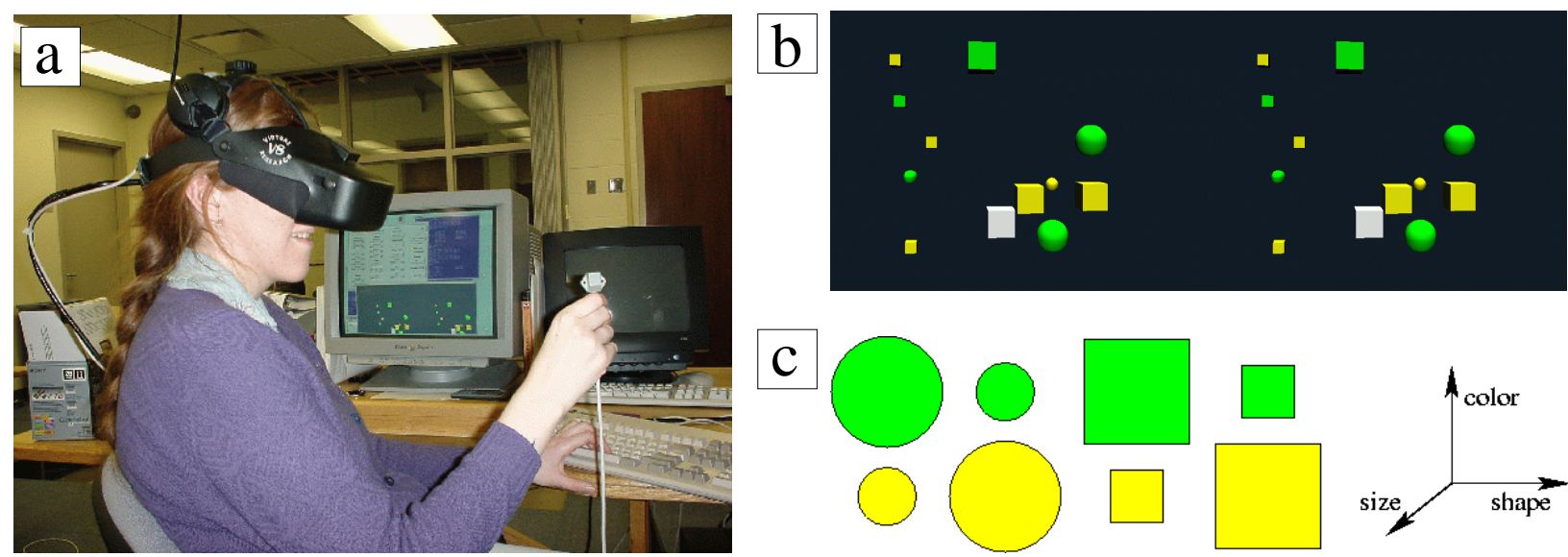

Figure 1: a: Subjects view the scene in a head mounted display. Responses are made by touching an object in virtual reality with a pointing device. b: Example of the stimulus. The views of left and right eye are shown next to each other. c: Objects are defined by the three attributes color, shape, and size, each of which can take two values. They can be thought of lying in a three dimensional space of possible objects.

of the twelve objects was randomly selected to be the object to be tracked. This object is referred to as the target; the remaining objects are referred to as distractors. Second, one of the visual attributes was randomly selected to be reliable. For each object, the value of the reliable attribute did not change during the course of a trial. In contrast, the other attributes were unreliable in the sense that their values were modified during a trial. For example, suppose that color was selected as the reliable attribute. The shapes and sizes of the target and distractor objects changed during a trial, but the colors of these objects did not.

A trial consisted of four phases. During the preparation phase the object to be tracked was uniquely defined by its white color. This phase ended after 3 seconds when the target turned either yellow or green. In the tracking phase all objects randomly changed the values of their unreliable attributes according to a Poisson process (i.e. at each time step there was a constant probability of an attribute changing its value). The parameter of the Poisson process was chosen so that an attribute changed its value within 0.3 seconds with probability 0.5 . The duration of the tracking phase was sampled from a uniform distribution ranging between 3 and 6 seconds. Note that, for the purpose of tracking the target, the informative properties of the target and distractors were the values of the reliable attribute, as well as their positions and velocities. If a subject lost track of the target, he or she could abort the trial by pressing a key on the keyboard. After the tracking phase, all objects turned invisible for 0.5 seconds. This occlusion phase simulated a short occlusion of the visual scene. Finally, in the response phase, two candidate objects were displayed on either side of the center of the environment, and subjects indicated which of the two they thought was more likely to have been the target object. Because the candidates were placed at a fixed location on each trial, subjects made their selection based on the candidates' visual attributes and not based on their spatial locations. Subjects indicated their selection by touching the candidate of their choice as quickly as possible with a three-dimensional pointing device. 
The two candidate objects had exactly two attribute values in common with the target at the end of the tracking phase, and one attribute value in common with each other. In other words, both candidates differed from the target's last appearance in one attribute, but this changed attribute was a different one for the two candidates. For example, if the target's last attribute values were (sphere, yellow, small), then the candidates' values may have been (sphere, yellow, big) and (cube, yellow, small). In this example, a subject's response would reflect whether the subject's cue integration strategy placed greater emphasis on the information provided by the shape cue or the size cue at the end of the trial.

Altogether there were nine types of trials: any of the three attributes could be the reliable one, and any of the three attributes could be the one whose value both candidates shared with the target at the end of the tracking phase. The nine types of trials were presented in random order forming a block. Half the subjects performed 10 blocks (90 trials) and the other half performed 20 blocks (180 trials). In two thirds of the trials, both candidates had the same value of an unreliable attribute as each other and as the target, meaning that they differed in the reliable attribute and an unreliable attribute. We call this set of trials reliable-unreliable trials. In the remaining trials, both candidates shared the reliable cue with the target, so that they differed in the two unreliable attributes. We call this set of trials unreliable-unreliable trials.

Subjects: The twenty subjects were students at the University of Rochester. They had normal or corrected-to-normal vision. They were naive to the purposes of the experiment.

\section{Results}

The experiment was designed to evaluate whether or not subjects quickly adapt their cue integration strategies on the basis of cue reliabilities. Our prediction was that subjects will quickly adapt their strategies so as to emphasize information provided by the reliable attribute and to discount information provided by unreliable attributes. We also predicted that subjects would tend to more equally weight the information provided by reliable and unreliable attributes when the value of an unreliable attribute had not changed for some significant duration of time.

In order to test these predictions, we limit our analysis to the set of reliable-unreliable trials, which comprised two thirds of all trials. It is useful for us to consider this set because it allows for a direct comparison of how much subjects based their decisions on the reliable attribute versus an unreliable attribute. As a matter of terminology, we refer to the unreliable attribute in which the two candidates differed as the 'relevant unreliable attribute.' In addition, we refer to the candidate with the same value of the reliable attribute as the target as the 'reliable-same candidate' and to the other candidate, the one with the same value of the relevant unreliable attribute as the target, as the 'unreliable-same candidate.'

The results of the analysis are shown in Figure 2. The horizontal axis gives the subject; the vertical axis gives the ratio of trials in which the subject chose the reliable-same candi- 

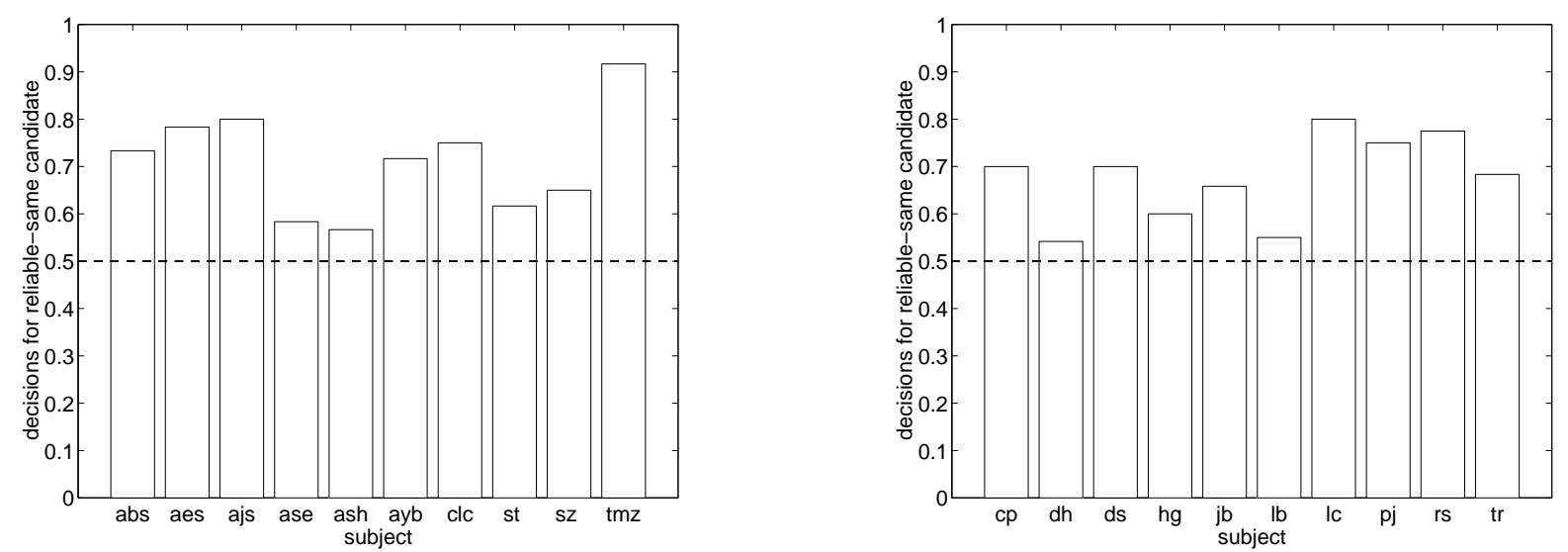

Figure 2: Ratio of decisions for the reliable-same candidate for different subjects. Left: 10 subjects who did 90 trials. Right: 10 subjects who did 180 trials.

date. If subjects' cue integration strategies were not sensitive to the reliability of cues, then subjects would be expected to have chosen the reliable-same candidate on 50 percent of the trials. However, subjects chose the reliable-same candidate significantly more often (mean $=69 \%$, standard error of the mean $=2.2 \%$ ) meaning that they rapidly adapted their cue integration strategies during an individual trial on the basis of attribute changes. Because the tracking phase of a trial lasted from 3 to 6 seconds, we can conclude that the temporal dynamics of the adaptation process is fast enough to be important at this time-scale.

In order to learn more about the temporal dynamics of the adaptation process, we considered how subjects' responses depended on the amount of time that the relevant unreliable attribute had been unchanged on the target before the simulated occlusion. For example, suppose that on two trials color was the relevant unreliable attribute, and that on the first trial the color of the target had not changed during the $200 \mathrm{msec}$ prior to the occlusion whereas on the second trial the color had not changed during the prior $2000 \mathrm{msec}$. We might reasonably expect that subjects will be more likely to chose the reliable-same candidate on the first trial because the relevant unreliable cue recently changed its value on this trial whereas on the second trial this cue had been relatively stable. Figure 3 shows that this is indeed the case. The horizontal axis gives the time in seconds that the relevant unreliable cue was unchanged prior to the occlusion; the vertical axis gives the average ratio of trials in which a subject chose the reliable-same candidate (the error bars give the standard error of the mean). The results are that subjects tended to emphasize the information provided by the reliable cue, and to discount the information provided by the relevant unreliable cue, when the relevant unreliable cue had changed soon before the occlusion. Importantly, when the relevant unreliable cue had not changed for 0.8 seconds or longer before the occlusion then subjects' performances were nearly at chance. In this case, they did not seem to distinguish between reliable and unreliable cues once the unreliable cue had been stable for this duration of time.

Another interesting aspect of the data is that it reveals that subjects' cue integration strategies were often biased toward emphasizing the information provided by a particular 


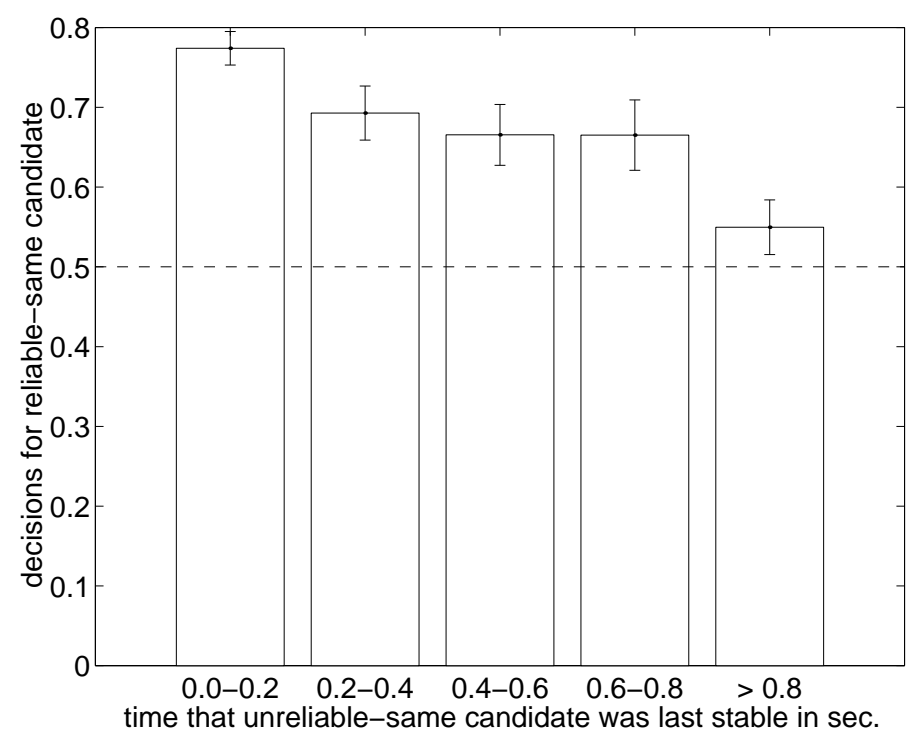

Figure 3: Ratio of decisions for the reliable-same candidate as a function of time that the unreliable-same candidate has last been stable.

cue. For each attribute, we counted the number of trials among all the experimental trials in which a subject chose a candidate object that had the same attribute value as the target object at the end of the tracking period. For example, if at the end of the tracking period the target was (sphere, yellow, small) and the subject chose a candidate that was (sphere, yellow, big), then we would increment the count of the shape and color attributes, but not the count of the size attributes. For 12 out of 20 subjects, the shape cue seemed to be emphasized. For 6 subjects the size cue was emphasized, and for 2 subjects the color cue was emphasized.

In the one third of trials constituting the unreliable-unreliable set, subjects choose between two candidates, which differ from the target in a different unreliable attribute. Generally, one of these two attributes has been stable for a longer amount of time. We tested, whether this influenced the subjects' decisions. Subjects chose the candidate that matches the target in the cue that has been stable a longer amount of time significantly more often (mean $59 \%$, SE of mean $1.8 \%, \mathrm{n}=20$ ). This result is consistent with our hypothesis of fast re-weighting of cues based on their reliability.

In order to gain insights into subjects' decision processes when choosing among the two candidates, we monitored the eye movements of two subjects doing the experiment. Eye tracking was done with a standard ASL eye tracking unit mounted in the head mounted display (Pelz et al., 1999). During the tracking phase of the experiment we found that subjects try to keep the target at the center of gaze by making saccadic eye movements. When subjects loose track of the object, they are usually aware of it and abort the trial. We considered the fixation patterns during the decision phase of the experiment. We categorized the fixation patterns into two classes (Tab. 1). At most once ( $88 \%$ of trials): The subject fixates each candidate at most once. Back and forth (12\% of trials): The subject looks 


\begin{tabular}{|l||c|c|c|}
\hline Pattern & all & stable/unstable & unstable/unstable \\
\hline \hline at most once & $143(88 \%)$ & $98(92 \%)$ & $45(80 \%)$ \\
\hline back and forth & $19(12 \%)$ & $8(8 \%)$ & $11(20 \%)$ \\
\hline
\end{tabular}

Table 1: Fixation patterns during the decision phase.

back and forth between the candidates fixating at least one candidate more than once. In both patterns, the subjects eventually fixate the candidate which they are selecting. We considered whether there is a difference in the relative frequency of fixation patterns for the two sorts of trials — the reliable-unreliable trials and the unreliable-unreliable trials. The result is summarized in Tab. 1. Interestingly, subjects used more fixations in the unreliableunreliable trials. The percentage of at most once patterns goes down while the percentage of the back and forth pattern increases. We regard this as an indication that the candidates are perceived as being more similar by the subjects in this condition. If so, this is consistent with the result that subjects put more emphasis on the reliable attribute, since if candidates do not differ in the emphasized reliable attribute, then subjects' will perceive them as more similar.

\section{Control Experiment}

Subject reports in the experiment suggested that a few subjects became aware of the fact that there is one reliable, i.e. unchanging, attribute in each trial and consciously tried to identify this attribute in each trial and chose in favor of it. We decided to consider a control experiment where all attributes are unreliable, preventing subjects from employing this strategy. Our prediction was that subjects would tend to favor the cue that has been stable for a longer amount of time, as suggested by the results from the unreliable-unreliable trials in the original experiment. Another difference to the first experiment regards the changes of the objects. Instead of following a Poisson process, changes occur as follows: Whenever a property changes, we randomly choose the time that this property is going to remain constant from a uniform distribution ranging from $0-2.5$ seconds. This modification ensured a higher percentage of trials where a cue has been stable for a relatively long amount of time. Ten subjects participated in the study. The result is that a subject's choice was in favor of the cue that had been stable for the longer time significantly more often (mean $55 \%, \mathrm{SE}$ of mean $2.2 \%, \mathrm{n}=10$ ). The effect is smaller than that for the unreliable-unreliable trials in the original experiment. This might be related to the different scheme of random attribute changes in this experiment. It allows subjects to predict the attribute changes to a certain extent. In particular, if an attribute has been stable for 2.5 seconds it is certain to change immediately. Hence, subjects may not strongly emphasize an attribute that has been stable for a long time since they can predict that it will change soon. Overall, our results are consistent with the original experiment. 


\section{Computational Model}

In order to better understand the temporal dynamics of the subjects' cue integration strategies, we developed a simple computational model that successfully accounts for subjects' responses.

Let $\mathcal{V}$ be a set whose elements are the possible attribute values: sphere, cube, yellow, green, small, and big. In addition, let $v \in \mathcal{V}$ denote an attribute value, and $f_{v}$ be a binary feature whose value is one if a candidate object has attribute value $v$; otherwise it is zero. For example, a candidate that is (sphere, yellow, small) would be indicated by the following feature values: $f_{\text {sphere }}=1, f_{\text {cube }}=0, f_{\text {yellow }}=1, f_{\text {green }}=0, f_{\text {small }}=1$, and $f_{\text {big }}=0$. We are interested in the probability, denoted $p(T)$, that a candidate object is the target. In our model, we assume that this probability is proportional to a weighted sum of the features:

$$
p(T) \propto \sum_{v \in \mathcal{V}} w_{v} f_{v}
$$

where $w_{v}$ is the weight of feature $v$ at the time of the decision. We assume that the weights are a product of two quantities, (i) a reliability $r_{v}$ that indicates how stable attribute value $v$ has been with respect to the target, and (ii) a bias $b_{v}$ that indicates the bias in a subject's cue integration strategy toward emphasizing information provided by attribute value $v$ :

$$
w_{v}=r_{v} b_{v}
$$

In regard to the temporal dynamics of the reliabilities $r_{v}$, we assume that these dynamics are given by a simple leaky integrator:

$$
\tau \dot{r}_{v}(t)=f_{v}(t)-r_{v}(t)
$$

where $t$ denotes time. In discrete-time, this equation may be re-written as

$$
r_{v}(t+\Delta t)=\gamma f_{v}(t)+(1-\gamma) r_{v}(t)
$$

where $\gamma=\frac{\Delta t}{\tau}$. A reliability $r_{v}$ is increased at time $t$ if the candidate has attribute value $v$ at time $t$; otherwise it is decreased. In regard to the biases $b_{v}$, we assume that these are constant values for a particular subject. In addition, we assume that the biases for attribute values of the same attribute are equal, and that the biases associated with the different attributes are a set of non-negative numbers that sum to one. For example, a possible

set of biases is $b_{\text {shape }} \equiv b_{\text {cube }}=b_{\text {sphere }}=0.5 ; b_{\text {color }} \equiv b_{\text {yellow }}=b_{\text {green }}=0.3$; and $b_{\text {size }} \equiv b_{\text {small }}=b_{\text {big }}=0.2$.

We denote a particular combination of features by $f^{i}$, where $f^{i}$ represents the set of all features $f_{v}^{i}, v \in \mathcal{V}$. For a particular feature combination $f^{0}, p\left(T \mid f^{0}\right)$ can be written as:

$$
p\left(T \mid f^{0}\right)=\frac{\sum_{v \in \mathcal{V}} r_{v} b_{v} f_{v}^{0}}{\sum_{c} \sum_{v \in \mathcal{V}} r_{v} b_{v} f_{v}^{c}},
$$


where the sum over $c$ runs over all possible feature combinations that an object can have. At the end of the trial the subject decides between two candidates whose feature combinations are denoted $f^{1}$ and $f^{2}$. Using Bayes rule with a prior probability of 0.5 for both candidates, we find that the probability of candidate 1 being the target, which we denote by $p(1)$, is given by:

$$
p(1)=\frac{p\left(T \mid f^{1}\right)}{p\left(T \mid f^{1}\right)+p\left(T \mid f^{2}\right)} \text {. }
$$

To use the model to predict subjects' responses, we exposed the model to the same trials as the subjects. In each trial, the model used Equation (4) during the tracking phase to update its estimates of the reliabilities. After the occlusion, the model was exposed to two candidate objects and it computed the probability that each candidate was the target. The model's prediction of a subject's response is the candidate with the larger probability of being the target.

The model has three free parameters: the time constant $\tau$ and two independent biases (recall that the biases sum to one). Based on a subject's responses, we performed an exhaustive search of the parameter space to find the parameter values that allowed the model to best fit the subject's responses in a maximum likelihood sense. Since we are considering a two alternative forced choice task, we compute the Bernoulli likelihood $L$ of a subject's decisions given a particular set of model parameters:

$$
L=\prod_{n=1}^{180} p_{n}(1)^{r_{n}} p_{n}(2)^{1-r_{n}}
$$

where $n$ indexes the trials, $p_{n}(i)$ is the probability of candidate $i$ being the target on trial $n$ as defined in (6), and $r_{n}$ describes the subject's response according to:

$$
r_{n}=\left\{\begin{array}{lll}
1 & : & \text { subject chose candidate } 1 \\
0 & : & \text { subject chose candidate } 2
\end{array} .\right.
$$

The time constant $\tau$ was evaluated between zero seconds and one second in steps of 0.05 seconds, and the biases were evaluated between zero and one in steps of 0.05 with the constraint that the three biases sum to one. For each possible setting of the parameter values we calculated the likelihood $L$ of a subject's responses. The parameter set which maximizes $L$ is the maximum likelihood estimate for that subject. In addition, we noted in how many trials the model predicted the subject's decision correctly with this set of parameters.

The results are shown in Figure 4 [for this analysis, only the ten subjects who ran in the long version of the experiment (180 trials) are included]. The horizontal axis gives the maximum likelihood estimate for the time constant $\tau$ for each subject; the vertical axis gives the percent of trials on which the model correctly predicts the subject's responses. On average, the model correctly predicts subjects' responses on $74 \%$ of the trials (standard deviation 8\%), whereas the chance level is 50\%. Given that the model is so simple (it is linear, assumes the attributes are independent, and uses a rudimentary form of temporal 


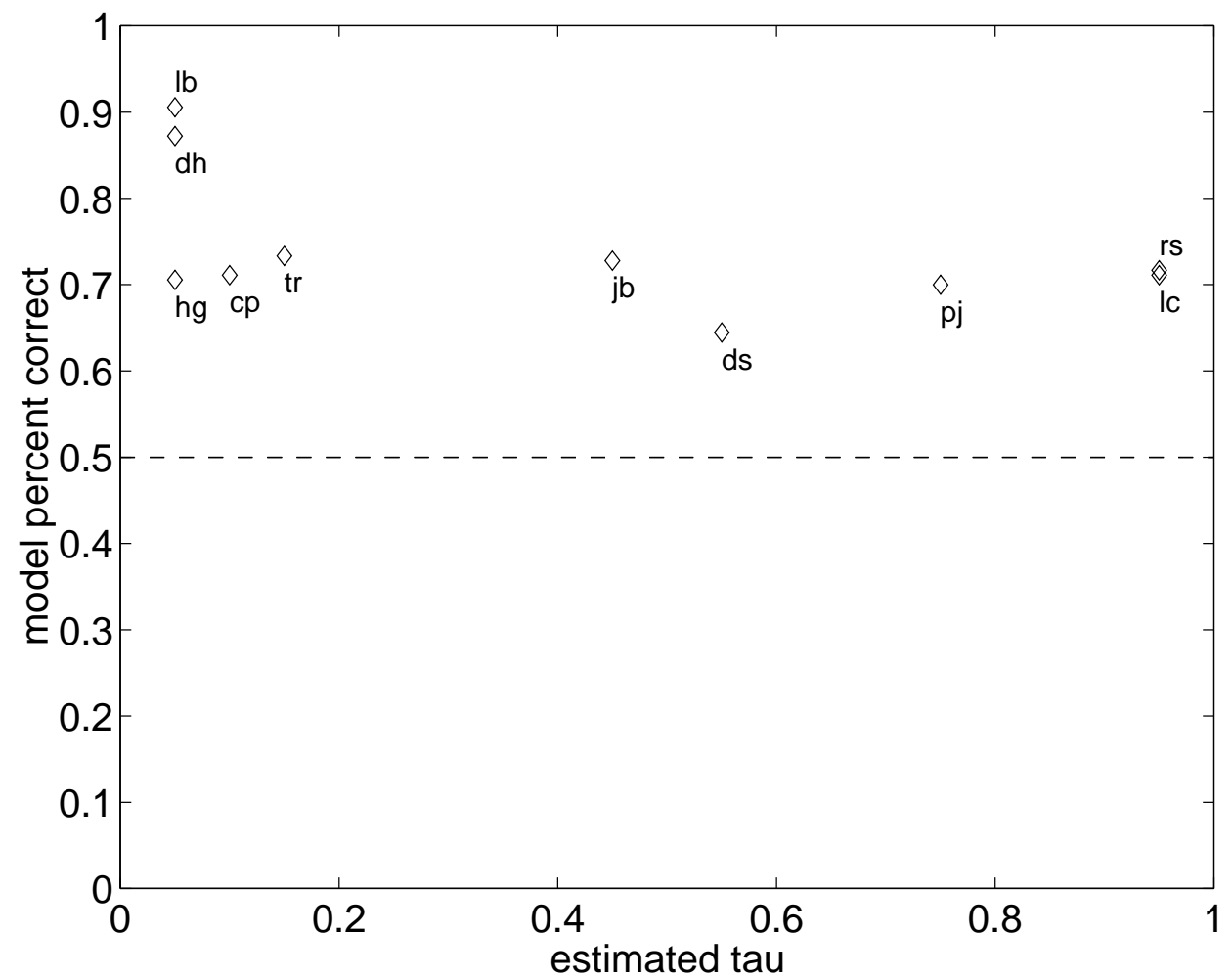

Figure 4: Results of the maximum likelihood estimation. The percentage of correctly predicted trials is given for different subjects. The horizontal axis marks the estimated time constant.

dynamics), we believe that this is a reasonably good fit between the model's predictions and the experimental data.

The time constants $\tau$ that are estimated for different subjects show considerable variability. To gain a better understanding of why the model estimated different time constants for different subjects, we performed several Monte Carlo simulations using the model. For this purpose, we created large sets of random stimuli and considered the decisions that the model would make for different time constants $\tau$. The results are summarized in Figure 5. These results are limited to the set of reliable-unreliable trials. The horizontal axis gives the time in seconds that the relevant unreliable cue was stable prior to the occlusion; the vertical axis gives the average ratio of trials in which the model chose the reliable-same candidate. The results show that as the value of $\tau$ gets larger, the model is more likely to chose the reliablesame candidate even when the relevant unreliable cue has been unchanged for a significant duration of time. Consequently, the percent of trials in which the reliable-same candidate is chosen is larger as $\tau$ gets larger. Thus, the differences between the estimated time constants for different subjects are related to the the different strength of the observed effect. The coefficient of correlation between the ratio of decisions for the reliable-same candidate and the estimated time constant $\tau$ is 0.86 (compare Figures 2 and 4 ). 


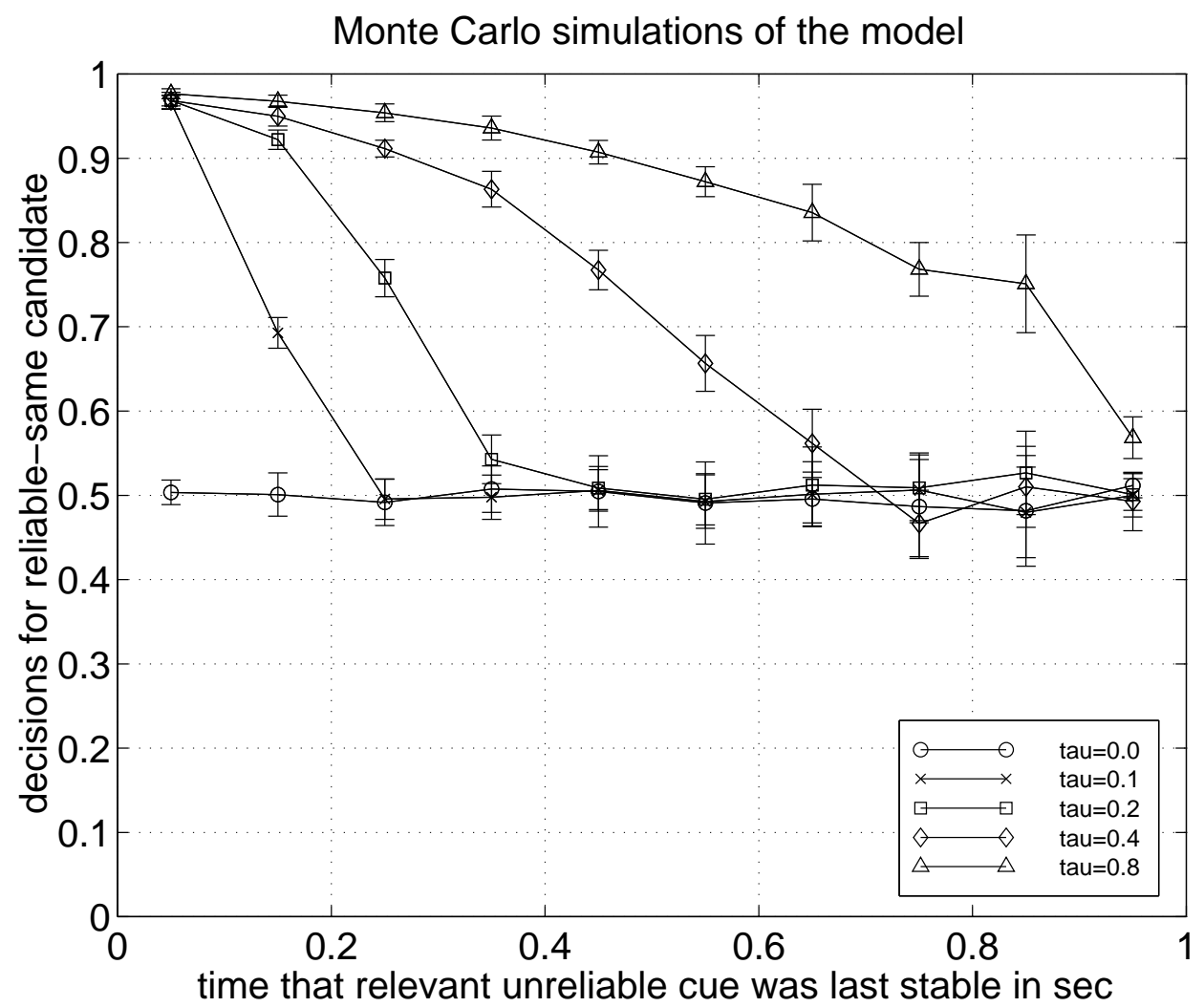

Figure 5: Monte Carlo study of the model. The ratio of trials where the stable-same candidate was selected is plotted as a function of how long the relevant unstable attribute has been stable before the occlusion for different model time constants $\tau$. Results are mean and standard deviations of 10 runs of 500 blocks of trials each. For the biases of the model we chose "typical" values estimated for subjects: $b_{\text {shape }}=0.5, b_{\text {color }}=0.2$, and $b_{\text {size }}=0.3$.

To determine whether or not our model with dynamic re-weighting fits subjects' data better than a static model we performed a Bayesian model comparison (MacKay, 1992). Model $M_{1}$ is the model introduced above and model $M_{2}$ is identical but with the time constant $\tau$ fixed at zero. Thus, model $M_{1}$ has three free parameters (2 independent biases and the time constant $\tau$ ), while model $M_{2}$ has only two free parameters (the biases). In the following, we refer to the parameter sets of $M_{1}$ and $M_{2}$ as $U_{1}$ and $U_{2}$, respectively. Using Bayesian statistics, the difference in complexity between the two models is properly accounted for (MacKay, 1992) during the comparison.

We are interested in the probability of $M_{i}(i=1,2)$ being the correct model given the data $D$ :

$$
p\left(M_{i} \mid D\right)=\frac{p\left(D \mid M_{i}\right) p\left(M_{i}\right)}{P(D)} .
$$




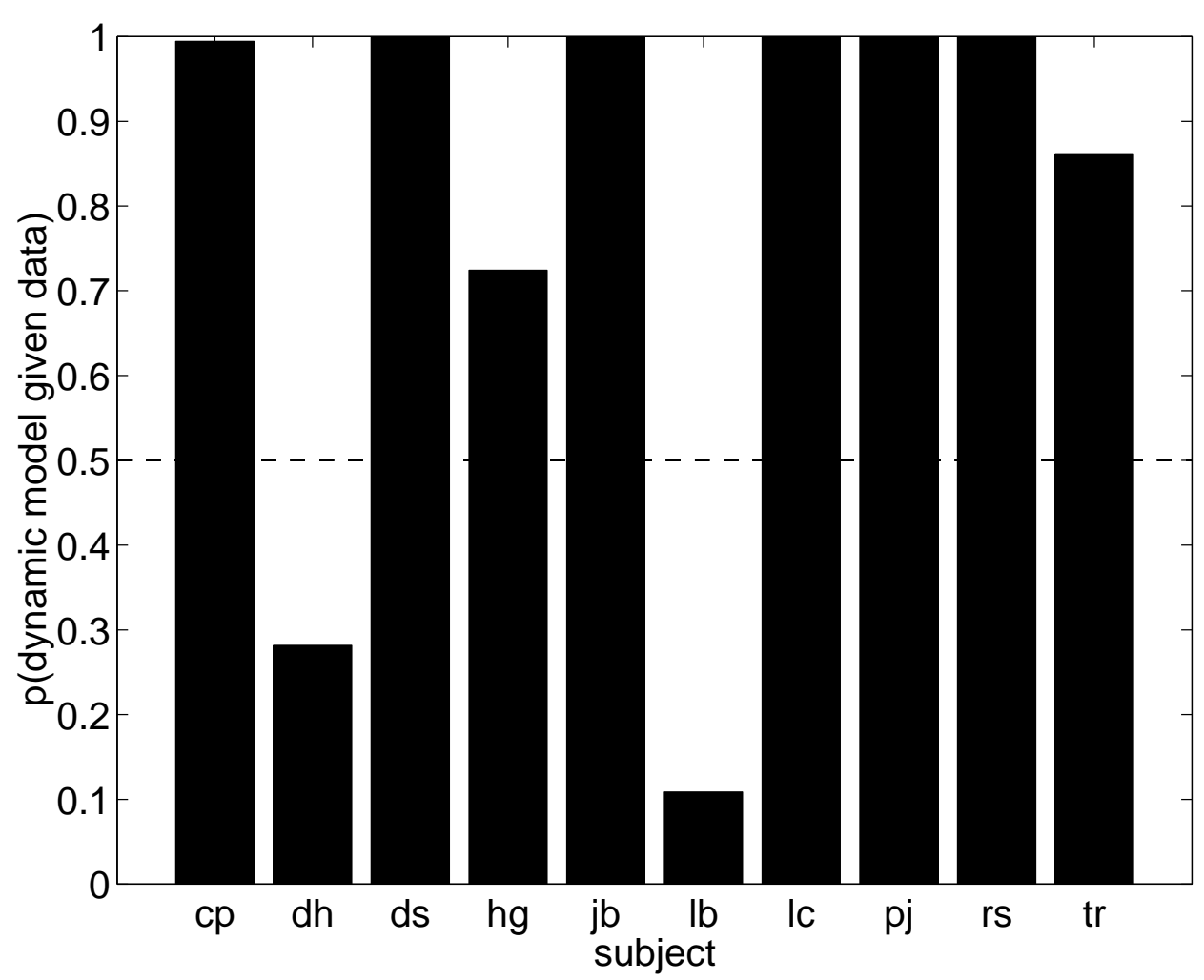

Figure 6: Comparison of the dynamic model M1 to the static model M2. The abscissa marks the subject; the ordinate gives the probability of M1 being the correct model.

Assuming uniform priors $p\left(M_{1}\right)=p\left(M_{2}\right)=1 / 2, P\left(M_{i} \mid D\right)$ is proportional to $p\left(D \mid M_{i}\right)$ since $p(D)$ does not depend on the model. We compute $p\left(D \mid M_{i}\right)$ by considering $p\left(D \mid M_{i}, U_{i}\right)$ and marginalizing over the parameters $U_{i}$ :

$$
p\left(D \mid M_{i}\right)=\sum_{U_{i}} p\left(D \mid M_{i}, U_{i}\right) p\left(U_{i}\right)
$$

Here, $p\left(U_{i}\right)$ is the prior probability distribution over the model parameters and $P\left(D \mid M_{i}, U_{i}\right)$ is just the likelihood defined in (7). By using uniform priors for $p\left(U_{i}\right)$ we can calculate $p\left(D \mid M_{i}\right)$ and hence $p\left(M_{i} \mid D\right)$, correctly taking into account that the models have a differing number of free parameters ${ }^{1}$. The results are shown in Figure 6. For eight out of ten subjects the dynamic model is clearly superior. For two subjects DH and LB, for whom the static model seems superior, it is interesting to note that (i) they were the subjects to show the least sensitivity to cue reliability (compare Figure 2) and (ii) they were the only ones to show extreme biases towards one particular cue. That is, these subjects tended to base their responses on one cue regardless of the relative reliabilities of the cues. We suggest that the strong bias towards one cue, which allows both the dynamic and the static model to predict their responses extremely well (compare Figure 4), overshadowed the dynamic

\footnotetext{
${ }^{1}$ The priors are also called Occam factors in the literature. The model with the smaller number of parameters has a bigger prior, in our case $p\left(U_{2}\right)>p\left(U_{1}\right)$.
} 
re-weighting of the cues. This suggestion was confirmed with Monte Carlo simulations that showed that a dynamic model with a strong bias towards one cue produces far fewer decisions for the reliable-same candidate than without a strong bias. Consequently, it is not surprising that subjects DH and LB chose the reliable-same candidate only slighthly more often than chance (see Figure 2). In summary, our analysis shows that the dynamic model is superior to the static model at describing the subjects' cue integration strategies and that temporal integration and rapid re-weighting of cues are necessary features for accounting for subjects' responses.

\section{Summary and Conclusions}

In summary, recent studies have demonstrated that visual cue integration strategies are adaptable in an experience-dependent manner. These studies have shown adaptation on a relatively long time-scale (hours or days), but have not considered the issue of whether or not adaptation can influence on-line processing by modifying observers' cue integration strategies on a short time-scale (fractions of a second). This article has studied the fast temporal dynamics of visual cue integration by reporting the results of an experiment using a tracking / identification task. Subjects tracked a target object among distractors and identified the target after an occlusion. Objects were defined by three visual attributes (color, shape, and size). Two of the attributes were unreliable in the sense that their values changed frequently within a trial, whereas the remaining reliable attribute was stable. The results are that subjects rapidly re-weighted the different cues on each trial by emphasizing the information provided by the reliable cue and by discounting the information provided by the unreliable cues. This re-weighting took place in less than one second and, thus, the results show that cue integration can exhibit adaptive phenomena on a very fast time-scale.

The experimental results were successfully modeled using a linear statistical model with simple temporal dynamics. It captures the main features of our data and is able to predict subjects' responses with reasonable accuracy. Future studies will need to develop more sophisticated models. We tested a model with different time constants for the shape, color, and size cues when trying to account for the data from our experiment, but this did not give significant advantages. However, our experiment was not designed to study differing time constants for different cues. A second potentially fruitful direction of research is to design a model that considers the distribution of cue values not just of the target object, but also of the distractors. Information provided by a cue that distinguishes the target from nearby distractors should be used more extensively than information provided by a cue that does not.

We expect the observed rapid re-weighting of cues to be a ubiquitous phenomenon, which affects sensory processing whenever cues change in their reliabilities or their inherent noise properties (Triesch, 2000), as is often the case in cue conflict experimental paradigms. Indeed, it might be the case that researchers have witnessed these effects earlier, but simply 
misinterpreted them as noise in the cue integration process. More experiments are needed to clarify these issues. 


\section{References}

Atkins, J. E., Fiser, J., \& Jacobs, R. A. (2000). Experience-dependent visual cue integration based on consistencies between visual and haptic percepts. Submitted for publication.

Bruno, N. \& Cutting, J. E. (1988). Minimodularity and the perception of layout. Journal of Experimental Psychology, 117, 161-170.

Ernst, M. O., Banks, M. S., \& Bülthoff, H. H. (2000). Touch can change visual slant perception. Nature Neuroscience, 3(1), 69-73.

Jacobs, R. A. \& Fine, I. (1999). Experience-dependent integration of texture and motion cues to depth. Vision Research, 39, 4062-4075.

Landy, M. S., Maloney, L. T., Johnston, E. B., \& Young, M. (1995). Measurement and modeling of depth cue combination: In defense of weak fusion. Vision Research, 35, $389-412$.

MacKay, D. J. C. (1992). Bayesian interpolation. Neural Computation, 4, 415-447.

Massaro, D. W. \& Friedman, D. (1990). Models of integration given multiple sources of information. Psychological Review, 97(2), 225-252.

Newman, E. A. \& Hartline, P. H. (1982). The infrared 'vision' of snakes. Sci. Amer., 246(3), $116-127$.

Pelz, J. B., Hayhoe, M. M., Ballard, D. H., Shrivastava, A., Bayliss, J. D., \& von der Heyde, M. (1999). Development of a virtual laboratory for the study of complex human behavior. In Proceedings of the SPIE - The International Society for Optical Engineering, Vol. 3639B, The Engineering Reality of Virtual Reality, San Jose, CA. SPIE.

Stein, B. \& Meredith, M. A. (1993). The Merging of te Senses. MIT Press.

Triesch, J. (2000). Democratic Integration: A theory of adaptive sensory integration. Technical Report NRL TR 00.1, Nat'l. Resource Lab'y. for the Study of Brain and Behavior, U. Rochester.

von Holst, E. (1950). Die Arbeitsweise des Statolithenapparates bei Fischen. Zeitschrift für vergleichende Physiologie, 32, 60-120.

Yuille, A. L. \& Bülthoff, H. H. (1996). Bayesian decision theory and psychophysics. In D. C. Knill \& W. Richards (Eds.), Perception as Bayesian Inference. New York: Cambridge University Press. 